\title{
Pregnancy After Treatment of. Ovarian Tumours
}

\author{
SHALINI RAJARAM, MONISHA GUPTA
}

Fertility-preserving surgery i.e. removing the affected ovary and preserving the contralateral ovary and the uterus followed by combination chemotherapy has become the standard of care in early stages and selected advanced ovarian GCTs. Stage IA epithelial ovarian cancers can also be treated by conservative surgery alone without adjuvant chemotherapy. Since 1989, bleomycin, etoposide, and cisplatin combination has become standard chemotherapy treatment for all ovarian GCTs. Three or four courses of chemotherapy have placed patients into remission with long-term survival. This, as the treatment of choice in younger women can conserve their reproductive function after effective treatment and has equivalent survival compared with bilateral salpingo-oophorectomy with or without hysterectomy.

Issues to be considered are return of menstruation and fertility after surgery and chemotherapy. Other issues would be pregnancy outcome both maternal and fetal. Fertility preserving surgery is usually advocated in premenarcheal girls in whom germ cell tumours are common and also in young post menarcheal girls. It was found that women treated before puberty have a reduced risk of developing gonadal damage relative to those treated after menarche. In one report from The M.D. Anderson Cancer Center, patients who developed menstrual dysfunction after completion of chemotherapy were significantly older at diagnosis than those who had normal menses (mean ages 17.7 and 13.6, respectively). ${ }^{1}$ The side-effects of chemotherapy especially

Department of Obstetrics \& Gynecology, University College of Medical Sciences \& Guru Teg Bahadur Hospital, Delhi.

E-mail : shalini.rajaram@labnetwork.com alkylating agents such as cyclophosphamide include gonadal failure. It has been seen that menarche occurs normally in premenarcheal girls and it takes about 6 months for return of fertility in post menarcheal girls. $80 \%$ of patients report normal return of menses. Premenarchal ovaries may be more resistant to the toxicity from the chemotherapy than postmenarchal ovaries because of the relatively larger amount of oocytes in reserve. This information is extremely important to parents of young girls diagnosed with malignant germ cell tumours of the ovary who are concerned about their daughters' future reproductive function. A small number of patients(3-5\%) have a risk of premature menopause.

The majority of the patients are placed on OCs during chemotherapy treatment. This is routinely recommended this to avoid any possible pregnancy during treatment with cytotoxic agents. Another legitimate concern of these young women, is what is the effect on their offspring after treatment with toxic agents at a young age. Treatment with mutagenic chemotherapeutic agents does not significantly increase the frequency of congenital anomalies ${ }^{2}$.

About $75-95 \%$ of women treated with fertility preserving surgery followed by chemotherapy succeed in achieving conception with or without treatment for infertility ${ }^{3,4,5}$. Pregnancy has also been reported in patients after treatment of advanced staged disease with eight of ten women with stage III disease conceiving ${ }^{3}$.

Thus most literature reviews suggests that fertility-preserving surgery followed by 
combination chemotherapy may not impede normal reproductive and menstrual function in women diagnosed with malignant germ cell tumours of the ovary. To date, children born to these patients have not experienced an increased risk for congenital malformations or developmental abnormalities. Fertilitypreserving surgery followed by appropriate chemotherapy should remain the standard of care even for women with malignant germ cell tumours of the ovary in advanced stages. Patients with this disease and their family members should be reassured about the high chance of retaining the patient's ability to conceive and have normal children after conservative surgery and combination chemotherapy.

\section{REFERENCES:}

1. Gershenson D. Management of early ovarian cancer: Germ cell and sex cord-stromal tumours. Gynecol Oncol 1994;55:S62-72.

2. Motte Rouge de La T, Pautier P, Duvillard P, Rey A, Morice P, Haie-Meder $C$, Kerbrat $P$ et al. Survival and reproductive function of 52 women treated with surgery and bleomycin, etoposide, cisplatin (BEP) chemotherapy for ovarian yolk sac tumour. Annals of Oncology 2008;19:1435-41.

3. Tangir $J$, Zelterman $G, M a W$, Schwartz PE. Reproductive function after conservative surgery and chemotherapy for malignant germ cell tumours of the ovary. Obstet Gynecol 2003;101:251-7.

4. Low JJH, Lewis CP, Crandon AJ, Hacker NF. Conservative surgery to preserve ovarian function in patients with malignant ovarian germ cell tumours. Cancer 2000;89:391-8.

5. Zanetta G, Bonazzi C, Cantu MG, Binidagger S, Locatelli $A$, Bratina $G$, et al. Survival and reproductive function after treatment of malignant germ cell ovarian tumours. J Clin Oncol 2001;19:1015-20. 